\title{
Above and beyond emotional suffering: the unique contribution of compassionate and uncompassionate self-responding in chronic pain
}

https://doi.org/10.1515/sjpain-2020-0082

Received May 30, 2020; accepted July 9, 2020; published online

August 25, 2020

\section{Abstract}

Objectives: Studies have shown that self-compassion plays a protective role against depression in women with chronic pain (CP). However, the majority of studies in CP have used the total score of the self-compassion scale (SCS), which have raised concerns due to potential overlap, not only between the uncompassionate self-responding factors and psychopathology, but also between self-compassion as a whole and other wellknown psychological processes (e.g., mindfulness, acceptance, psychological flexibility). This calls for a more nuanced understanding of which components of (un)compassionate self-responding adds to better mental health in CP.

Methods: This study explores the unique contribution of compassionate and uncompassionate self-responding to depressive symptoms in women with $\mathrm{CP}$ undergoing pain consultation $(\mathrm{n}=49)$.

Results: Correlation analyses suggest that compassionate self-responding only significantly correlates with progress in valued living, while the uncompassionate selfresponding significantly correlates with pain fusion, pain avoidance, obstructions to valued living and depression.

*Corresponding author: Sérgio A. Carvalho, CINEICC, Faculdade de Psicologia e de Ciências da Educação da Universidade de Coimbra, Rua do Colégio Novo, Apartado 6153, 3001-802, Coimbra, Portugal, Phone: +351 239 851450, Fax: +351239 851462,

E-mail: sergiocarvalho@fpce.uc.pt

José Pinto-Gouveia and Paula Castilho: Cognitive-Behavioural Research Centre (CINEICC), Faculty of Psychology and Educational Sciences, University of Coimbra, Coimbra, Portugal

David Gillanders: School of Health in Social Sciences, University of Edinburgh, Edinburgh, UK

Teresa Lapa, Ana Valentim, Elsa Santos and Juliana Paciência: Anaesthesiology Service, Coimbra Hospital and University Centre, Coimbra, Portugal
Multiple regression analysis showed that self-compassion contributes to depressive symptoms $\left(\mathrm{R}^{2}=8 \%\right)$ above and beyond pain intensity and disability $\left(\mathrm{R}^{2}=12 \%\right)$ and psychological (in) flexibility processes $\left(\mathrm{R}^{2}=31 \%\right)$, and uncompassionate (but not compassionate) self-responding uniquely contributes to depressive symptoms $\left(s r^{2}=18 \%\right)$. Conclusions: Findings suggest that uncompassionate selfresponding is a stronger contributor to depression in $\mathrm{CP}$ than compassionate self-responding. Clinical implications are further discussed.

Keywords: chronic pain; depression; pain disability; psychological inflexibility; self-compassion.

\section{Introduction}

Chronic Pain (CP) is an impactful medical condition characterized by constant or sporadic pain or discomfort for at least 3 months [1], with great impact on functioning (e.g., [2]). It is well-established the role of psychological processes in the etiology of CP symptomatology, in particular their impact on depressive symptoms (e.g., [3]).

Self-compassion is described as the ability to be sensitive to personal suffering and motivated to courageously alleviate it [4, 5], and it has been theoretically proposed [6] and empirically suggested [7] to be beneficial in CP, even when it is not a direct focus of a psychological flexibility program [8]. Indeed, self-compassion seems to be a crosssectional predictor of depressive symptoms [9], of negative affect and pain disability [10], and to prospectively predict depressive symptoms [11]. Nevertheless, a more in depth analysis of the role of self-compassion is needed to better understand its unique role in $\mathrm{CP}$, particularly comparatively to other related psychological processes. Also, there seems to be an ongoing discussion on which subcomponent(s) of self-compassion contribute to the beneficial or detrimental effect of the overall construct. For 
example, one study found that the role of self-compassion depended on how its widely used measure [SelfCompassion Scale; four] was examined: while the total score was strongly correlated to depression, its association was weak when considering exclusively the compassionate self-responding subcomponents (self-kindness, common humanity, mindfulness) rather than the uncompassionate self-responding ones (self-judgment, isolation, over-identification) [12]. This taps into the fact that a great portion of the studies on self-compassion in CP have only been focused on self-compassion as a whole, rather than exploring its subcomponents and providing a more finetuned picture of its benefits. This is particularly relevant when considering the potential overlap of self-compassion and related psychological processes such as psychological (in)flexibility, acceptance and mindfulness (for a topical discussion, see [13]). For example, one study found that the subcomponents self-judgment, isolation and overidentification load into a fusion/avoidance psychological inflexibility process, and common humanity and mindfulness load into an underlying present moment awareness process [14]. Another recent study explored the role of selfcompassion in depression in $\mathrm{CP}$, but did not report the unique contribution of its subcomponents nor controlled for the effect of related constructs [15]. It is particularly underexplored the relationship between self-compassion and valued living, even though tentative data seem to corroborate it by showing that self-compassion is related to the behavioral component of acceptance of pain [16]. Indeed, due to potential conceptual overlap, it is important to explore the subcomponents of self-compassion while controlling for psychological processes related to acceptance, valued action and present moment awareness [14], given that these also seem to contribute to depression in $\mathrm{CP}$ (e.g., [17]).

The current study aims to explore the unique role of the subcomponents of self-compassion in depressive symptoms in CP. We hypothesize that uncompassionate selfresponding, but not compassionate self-responding, is a significant predictor of depression, above and beyond pain disability, fusion and avoidance, and valued living.

\section{Methods}

The current study was conducted in a sample of women with musculoskeletal CP $(n=49)$ who were undergoing pain management consultation in a Portuguese healthcare unit. The physician assessed inclusion criteria (1. having CP; 2. age $>18$ years) and invited eligible patients to participate, informed about anonymity of data and assured the voluntary nature of the study. Then a clinical psychologist assessed for exclusion criteria (1. severe clinical depression; 2. psychosis; 3. non- suicidal self-injury; 4. suicide attempt(s) in the last 6 months; 5 . substance abuse) using an adapted version of the SCID-1. The current sample had a mean age of $50.49(\mathrm{SD}=7.69)$. The majority were married $(n=33 ; 67.3 \%)$ and were currently employed $(n=31 ; 66.0)$. The majority of participants had a primary education ( $\mathrm{n}=29 ; 59.2 \%)$, some had a high school degree $(\mathrm{n}=12 ; 24.5 \%)$ and others a bachelors or higher education degree $(n=8 ; 16.3 \%)$. The majority had fibromyalgia $(n=25 ; 55.6 \%)$ and/or low back pain $(n=16 ; 35,6 \%)$ and/or other $(n=21 ; 46.7 \%)$, were taking more than two medications for $\mathrm{CP}(\mathrm{n}=43 ; 87.8 \%)$, of which 24 (48.98\%) were taking opioids, and some had other chronic illnesses $(\mathrm{n}=17 ; 34.7 \%)$, such as type-II diabetes $(\mathrm{n}=3 ; 17.6 \%)$, hypothyroidism $(\mathrm{n}=2 ; 11.8 \%)$, chronic gastritis $(\mathrm{n}=2 ; 11.8 \%)$, and/or other $(\mathrm{n}=10 ; 59 \%)$. The study was previously approved by the Ethics Committee of the Faculty of Psychology and Educational Sciences of the University of Coimbra.

Pain intensity was assessed with Numeric Pain Rating Scale (NPRS; [18]), which measures pain intensity on a 11-point scale ( 0 = "No pain"; $10=$ "Worst imaginable pain"). A composite was calculated with the mean of (1) pain currently experienced, (2) lowest pain in last $24 \mathrm{~h}$, (3) highest pain in last $24 \mathrm{~h}$. Pain disability was assessed with the Pain Disability Index (PDI; [19]), which measures on an 11-point scale ( 0 = no disability; $10=$ worst disability) the degree of disruption in seven daily life caused by pain. Depressive symptoms were measured with the Depression, Anxiety and Stress Scale 21-items version (DASS-21; [20]). For the purpose of this study, only the depression subscale was considered, which is composed of 7 -items assessed on a 4-point scale $(0=$ did not apply to me at all; 3 = applied to me very much or most of the time). Pain fusion and pain avoidance was assessed through Psychological Inflexibility in Pain Scale (PIPS; [21]), which measures pain-related psychological inflexibility on a 7-point scale ( 1 = never true; 7 = always true), with higher scores suggesting higher pain fusion and avoidance. Valued living was assessed with the Valuing Questionnaire (VQ; [22]), composed of 10-items that measure obstacles to and progress in valued living on a 7-point scale ( 0 = not at all true; 6 = completely true). Self-compassion was assessed with the Self-Compassion Scale (SCS; [4]). It measures six domains of compassionate self-responding (self-kindness, common humanity and mindfulness) and uncompassionate self-responding (self-judgment, isolation, over-identification). It is composed of 26-items assessed on a 5-point scale ( 1 = almost never; 5 = almost always). The psychometrics of all measures has been examined in chronic pain samples. The Portuguese validated versions of each scale were used.

All statistical analyses were conducted using SPSS statistics software version 23.0 (IBM corp., 2011). Pearson's correlation coefficients were calculated to examine patterns of association between the self-compassion scale components, pain-related and depressive symptoms, and psychological (in)flexibility processes between variables in study. Hierarchical regression analyses were performed to explore which components of the SCS uniquely contributed (unique variance: $s r^{2}$ ) to depressive symptoms in CP above and beyond pain disability and psychological (in) flexibility processes. This was examined by progressively adding to the model variables in four steps: step (1) pain disability; step (2) pain fusion and avoidance; step (3) obstacles and progress in valued living; step (4) self-compassion.

\section{Results}

Results from correlation analysis showed that the uncompassionate self-responding was significantly correlated 
Table 1: Means, Standard Deviations and Intercorrelations between variables $(n=49)$.

\begin{tabular}{|c|c|c|c|c|c|c|c|c|c|c|c|c|}
\hline Variables & M & SD & $\alpha$ & 1 & 2 & 3 & 4 & 5 & 6 & 7 & 8 & \\
\hline 1. Compassionate self-responding & 40.77 & 6.88 & 0.80 & - & - & - & - & - & - & - & - & \\
\hline 2. Uncompassionate self-responding & 40.98 & 10.45 & 0.92 & $-0.22^{\mathrm{ns}}$ & - & - & - & - & - & - & - & \\
\hline 3. Pain fusion & 37.18 & 5.65 & 0.82 & $-0.04^{\mathrm{ns}}$ & $0.48^{* *}$ & - & - & - & - & - & - & \\
\hline 4. Pain avoidance & 52.59 & 12.51 & 0.92 & $-0.03^{\text {ns }}$ & $0.62^{* \star *}$ & $0.67^{* \star *}$ & - & - & - & - & - & \\
\hline 5. Obstructions to valued living & 17.18 & 8.36 & 0.85 & $-0.17^{\mathrm{ns}}$ & $0.62^{* * *}$ & $0.38^{* *}$ & $0.59^{* * *}$ & - & - & - & - & \\
\hline 6. Progress in valued living & 21.28 & 6.33 & 0.81 & $0.33^{*}$ & $-0.09^{\mathrm{ns}}$ & $-0.24^{\mathrm{ns}}$ & $-0.19^{\mathrm{ns}}$ & $-0.32^{*}$ & - & - & - & \\
\hline 7. Pain intensity & 6.32 & 1.52 & 0.87 & $0.05^{\mathrm{ns}}$ & $0.24^{\mathrm{ns}}$ & $0.42^{* *}$ & $0.25^{\mathrm{ns}}$ & $0.23^{\mathrm{ns}}$ & $-0.04^{\mathrm{ns}}$ & - & - & \\
\hline 8. Pain disability & 39.67 & 11.54 & 0.86 & $0.19^{\text {ns }}$ & $0.28^{\text {ns }}$ & $0.28^{*}$ & $0.41^{* *}$ & $0.32^{*}$ & $0.06^{\mathrm{ns}}$ & $0.41^{* *}$ & - & \\
\hline 9. Depressive symptoms & 8.65 & 5.44 & 0.89 & $-0.24^{\mathrm{ns}}$ & $0.70^{\star \star *}$ & $0.45^{* *}$ & $0.61^{* * *}$ & $0.67^{* \star *}$ & $-0.35^{*}$ & $0.24^{\mathrm{ns}}$ & $0.30^{*}$ & \\
\hline
\end{tabular}

$\mathrm{p}<0.05$.

$* 0.01$

$* 0 \times 0.001$.

ns $=$ non-significant. Bold values indicate statistical significance.

with psychological inflexibility in pain, with obstructions to valued living and depression. Pain intensity was correlated with fusion with pain and pain disability. Compassionate self-responding only significantly correlated with progress in valued living. Pain intensity only correlated significantly with pain fusion. Pain disability correlated significantly with psychological (in)flexibility processes, except progress in valued living (see Table 1).

To better understand the unique contribution of the SCS components to depressive symptoms, hierarchical regression analyses were conducted (see Table 2).

Results showed that depressive symptoms were only significantly predicted by the uncompassionate selfresponding component of the SCS (unique variance: $s r^{2}=0.18$ ) above and beyond pain intensity and disability, pain fusion and avoidance, and obstruction and progress to valued living. It should be noted that the SCS contributes $8 \%$ to the variance of depressive symptoms, above and beyond pain intensity and disability $\left(\mathrm{R}^{2}=12 \%\right)$, pain fusion and avoidance $\left(R^{2}=31 \%\right)$ and valued living $\left(R^{2}=13 \%\right)$.

\section{Discussion}

A pattern of associations seems to suggest that the uncompassionate self-responding subcomponent of the SCS (self-judgment, isolation, over-identification), and not the compassionate self-responding (self-kindness, common humanity, mindfulness), is significantly correlated to depressive symptoms. It should be noted that the current sample did not present clinically severe levels of depression, given that severe depression was an exclusion criteria. This warrants careful interpretation of results and extrapolation for severely depressed CP patients. Nonetheless, results seems to echo previous results that suggested that the uncompassionate self-responding subcomponents of the SCS are the ones greatly contributing to the effect of SCS [12]. Interestingly, while uncompassionate self-responding was the one correlated with obstructions in valued living, it was compassionate self-responding that correlated with progress in valued living, which seems to align with the assertion that compassion has an actionorientation $[5,16]$. Particularly in $\mathrm{CP}$, this calls for a larger discussion and further empirical analysis of whether selfcompassionate programs in $\mathrm{CP}$ operate through an increase in self-compassionate attributes (increase in selfkindness, the acknowledgment of suffering as part of the common humanity, and a mindful stance) or rather a decrease of self-directed judgment, feelings of isolation and an over-identification with personal difficulties and suffering. Indeed, it seems that one study found that it does increase self-compassionate attributes in women with fibromyalgia [7], however it did not control for the effect of the uncompassionate self-responding attributes. When examining the unique contribution of the SCS components, uncompassionate self-responding emerged as the only significant predictor of depressive symptoms, above and beyond pain intensity and disability and (in)flexibility processes, suggesting that pain management programs that aim to decrease depressive symptoms would benefit from focusing on the specific uncompassionate subcomponents (self-judgment, but also feelings of isolation and over identification). This should be interpreted with caution, given that a more comprehensive analysis, with larger sample sizes, could provide evidence that compassionate self-responding indeed impacts on depressive symptoms not directly, but through other processes, such as behavior activation and/or commitment to valued action. Nonetheless, these results corroborate previous results showing that the SCS significantly predicts depressive 
Table 2: Hierarchical multiple regression analysis of predictors of depressive symptoms $(n=49)$.

\begin{tabular}{|c|c|c|c|c|}
\hline Blocks & $\mathbf{R}^{2}$ & $\Delta \mathbf{R}^{2}$ & $\beta^{(\text {(p-Value) }}$ & $\begin{array}{r}\text { Unique } \\
\text { variance } s r^{2}\end{array}$ \\
\hline $\begin{array}{l}\text { Step } 1 \text {. Pain intensity and } \\
\text { disability }\end{array}$ & 0.12 & 0.12 & & \\
\hline Pain intensity & & & $0.14^{(0.39)}$ & 0.02 \\
\hline Pain disability & & & $0.27^{(0.10)}$ & 0.07 \\
\hline $\begin{array}{l}\text { Step 2. Pain fusion and } \\
\text { avoidance }\end{array}$ & 0.44 & 0.31 & & \\
\hline Pain intensity & & & $-0.01^{(0.96)}$ & 0.00 \\
\hline Pain disability & & & $0.08^{(0.60)}$ & 0.01 \\
\hline Pain fusion & & & $0.16^{(0.36)}$ & 0.02 \\
\hline Pain avoidance & & & $0.50^{(0.01)}$ & 0.17 \\
\hline Step 3. Valued living & 0.56 & 0.13 & & \\
\hline Pain intensity & & & $-0.02^{(0.85)}$ & 0.00 \\
\hline Pain disability & & & $-0.04^{(0.78)}$ & 0.00 \\
\hline Pain fusion & & & $0.13^{(0.45)}$ & 0.02 \\
\hline Pain avoidance & & & $0.29^{(0.11)}$ & 0.07 \\
\hline Values obstruction & & & $0.40^{(0.01)}$ & 0.17 \\
\hline Values progress & & & $-0.09^{(0.46)}$ & 0.02 \\
\hline Step 4. Self-compassion & 0.65 & 0.08 & & \\
\hline Pain intensity & & & $-0.01^{(0.96)}$ & 0.00 \\
\hline Pain disability & & & $0.03^{(0.81)}$ & 0.00 \\
\hline Pain fusion & & & $-0.03^{(0.87)}$ & 0.00 \\
\hline Pain avoidance & & & $0.19^{(0.27)}$ & 0.04 \\
\hline Values obstruction & & & $0.24^{(0.11)}$ & 0.07 \\
\hline Values progress & & & $-0.16^{(0.19)}$ & 0.05 \\
\hline $\begin{array}{l}\text { Compassionate self- } \\
\text { responding }\end{array}$ & & & $0.02^{(0.84)}$ & 0.00 \\
\hline $\begin{array}{l}\text { Uncompassionate self- } \\
\text { responding }\end{array}$ & & & $-0.44^{(0.01)}$ & 0.18 \\
\hline
\end{tabular}

Pain Disability = Pain Disability Index; Pain Fusion and Avoidance $=$ Psychological Inflexibility in Pain Scale; Valued living $=$ Valuing Questionnaire; Self-compassion $=$ Self-Compassion Scale. Bold values indicate statistical significance.

symptoms in CP (e.g., $[11,15])$, and furthers the results by examining the unique contribution of its subcomponents while controlling for intensity, disability and psychological (in)flexibility processes [14]. When looking thoroughly to the uncompassionate items of the SCS (particularly the isolation ones), it seems clear that decreasing uncompassionate self-responding does not merely equate to promoting behavioral activation nor valued based action, but rather decreasing a sense of social disconnection, downward comparative criticism and feelings of failure when experiencing setbacks. However, these results should be interpreted with caution due to study limitations. For one, this study should be replicated in a larger sample, which would allow the test more complex models that would provide a much thorough analysis. Also, the all-female sample does not allow generalization to other genders. In fact, there seems to be gender differences in selfcompassion (men reported to be more self- compassionate) [23], but to our knowledge this have never been explored in CP. Additionally, the relatively low levels of depressive symptoms does not allow generalization of these results to participants with severe depression. Finally, the cross-sectional nature of the study does not allow for causality. Future studies should consider experimental and longitudinal designs to establish causality and/or temporal relationships between variables. Nevertheless, the current study contributes to a better understanding of self-compassion in $\mathrm{CP}$ that might inform psychological acceptance-, mindfulness- and compassionate-based pain management programs. It provides evidence for the importance of focusing on uncompassionate self-responding not exclusively through increasing self-kindness, mindfulness and common humanity, but also by promoting feelings of safeness, connectedness and belongingness. Indeed, it seems that the focus should be not only on decreasing selfjudgment, but also on helping deactivate threat-focused feelings of isolation [5] that may result from a sense of separateness and disconnection due to pain-focused internal experiences. Compassion-based exercises to $\mathrm{CP}$ may benefit not only from focusing on acceptance and self-kindness, but also from developing feelings of overall connectedness and safeness.

Research funding: Research by the first author (Sérgio A. Carvalho) is supported by a Ph.D. Grant (SFRH/BD/112833/ 2015), sponsored by FCT (Portuguese Foundation for Science and Technology).

Author contributions: All authors have accepted responsibility for the entire content of this manuscript and approved its submission.

Competing interests: Authors state no conflict of interest. Informed consent: Informed consent was obtained from all individual adult participants included in the study.

Ethical approval: All procedures performed in studies involving human participants were in accordance with the ethical standards of the institutional research committee and with the 1964 Helsinki Declaration and its later amendments or comparable ethical standards.

\section{References}

1. Merksey H, Bogduk N. Classification of chronic pain-descriptions of chronic pain syndromes and definitions of pain terms, 2 nd ed. Seattle: IASP Press; 1994.

2. Breivik H, Eisenberg E, O'Brien T. The individual and societal burden of chronic pain in Europe: the case for strategic prioritisation and action to improve knowledge and availability of appropriate care. BMC Publ Health 2013;13:1229. 
3. Gatchel RJ, Peng YB, Peters ML, Fuchs PN, Turk DC. The biopsychosocial approach to chronic pain: scientific advances and future directions. Psychol Bull 2007;133:581-624.

4. Neff KD. The development and validation of a scale to measure self-compassion. Self Ident 2003;2:223-50.

5. Gilbert P. Compassion: conceptualisations, research and use in psychotherapy. New York/London: Routledge; 2005.

6. Purdie F, Morley S. Compassion and chronic pain. Pain 2016;157: 2625-7.

7. Montero-Marin J, Van Gordon W, Shonin E, Navarro-Gil M, Gasión V, López-del-Hoyo Y, et al. Attachment-based compassion therapy for ameliorating fibromyalgia: mediating role of mindfulness and self-compassion. Mindfulness 2020;11:816-28.

8. Vowles KE, Witkiewitz K, Sowden G, Ashworth J. Acceptance and commitment therapy for chronic pain: evidence of mediation and clinically significant change following an abbreviated interdisciplinary program of rehabilitation. J Pain 2014;15:101-13.

9. Costa J, Pinto-Gouveia J. Experiential avoidance and selfcompassion in chronic pain. J Appl Psychol 2013;43:1578-91.

10. Wren AA, Somers TJ, Wright MA, Goetz MC, Leary MR, Fras AM, et al. Self-compassion in patients with persistent musculoskeletal pain: relationship of self-compassion to adjustment to persistent pain. J Pain Symptom Manag 2012;43: 759-70.

11. Carvalho SA, Trindade IA, Gillanders D, Pinto-Gouveia J, Castilho $P$. Self-compassion and depressive symptoms in chronic pain (CP): a 1-year longitudinal study. Mindfulness 2020;11:709-19.

12. López A, Sanderman R, Schroevers MJ. A close examination of the relationship between self-compassion and depressive symptoms. Mindfulness 2018. https://doi.org/10.1007/s12671018-0891-6.

13. Neff K, Tirch D. Self-compassion and ACT. In: Kashdan TB, Ciarrochi J, editors. The context press mindfulness and acceptance practica series. Mindfulness, acceptance, and positive psychology: the seven foundations of well-being.
Oakland, CA: Context Press/New Harbinger Publications; 2013: pp. 78-106.

14. Vowles KE, Sowden G, Ashworth J. A comprehensive examination of the model underlying acceptance and commitment therapy for chronic pain. Behav Ther 2014;45:390-401.

15. Edwards KA, Pielech M, Hickman J, Ashworth J, Sowden G, Vowles $\mathrm{KE}$. The relation of self-compassion to functioning among adults with chronic pain. Eur J Pain 2019;23:1538-47.

16. Carvalho SA, Gillanders D, Palmeira L, Pinto-Gouveia J, Castilho P. Mindfulness, selfcompassion, and depressive symptoms in chronic pain: the role of pain acceptance. J Clin Psychol 2018;74: 2094-106.

17. Scott W, McCracken LM, Norton S. A confirmatory factor analysis of facets of psychological flexibility in a sample of people seeking treatment for chronic pain. Ann Behav Med 2016;50:285-96.

18. Hartrick CT, Kovan JP, Shapiro S. The numeric rating scale for clinical pain measurement: a ratio measure?. Pain Pract 2003;3: 310-16.

19. Pollard CA. Preliminary validity study of the pain disability index. Percept Mot Skills 1984;59:974.

20. Lovibond PF, Lovibond SH. The structure of negative emotional states: comparison of the depression anxiety stress scales (DASS) with the beck depression and anxiety inventories. Behav Res Ther 1995;33:335-43.

21. Wicksell RK, Renöfält J, Olsson GL, Bond FW, Melin L. Avoidance and cognitive fusion-central components in pain related disability? development and preliminary validation of the psychological inflexibility in pain scale (PIPS). Eur J Pain 2008;12: 491-500.

22. Smout M, Davies M, Burns N, Christie A. Development of the valuing questionnaire (VQ). J Contextual Behav Sci 2014;3:16472.

23. Yarnell LM, Stafford RE, Neff KD, Reilly ED, Knox MC, Mullarkey M. Meta-analysis of gender differences in self-compassion. Self Ident 2015;14:499-520. 